Highlights (for review)

Highlights

Simulation of bispyridinium compounds at human nAChRs reveals interaction sites

Longer bispyridiniums can also interact at an allosteric site

Two-electrode voltage clamp recordings confirm the simulations 


\section{Molecular determinants of binding of non-oxime bispyridinium nerve agent antidote compounds to the adult muscle nAChR}

Max Epstein ${ }^{1}$, Karan Bali ${ }^{1}$, Thomas J. Piggot ${ }^{2}$, A. Christopher Green ${ }^{2}$, Christopher M. Timperley ${ }^{2}$, Mike Bird $^{2}$, John E. H. Tattersall ${ }^{2}$, Isabel Bermudez ${ }^{3}$ and Philip C. Biggin ${ }^{1^{*}}$

${ }^{1}$ Department of Biochemistry, Structural Bioinformatics and Computational Biochemistry, University of Oxford, Oxford, UK

${ }^{2}$ Chemical, Biological and Radiological Sciences Division, Defence Science and Technology Laboratory (DSTL), Porton Down, Salisbury, Wiltshire, UK

${ }^{3}$ Department of Biological and Medical Sciences, Faculty of Health and Life Sciences, Oxford Brookes University, Oxford, UK

*To whom correspondence should be addressed.

Philip.biggin@bioch.ox.ac.uk

Keywords: nicotinic acetylcholine receptor, nerve agent, antidote, molecular dynamics, simulation, allosteric. 


\begin{abstract}
Organophosphorus nerve agents (NAs) are the most lethal chemical warfare agents and have been used by state and non-state actors since their discovery in the 1930s. They covalently modify acetylcholinesterase, preventing the breakdown of acetylcholine (ACh) with subsequent loss of synaptic transmission, which can result in death. Despite the availability of several antidotes for OPNA exposure, none directly targets the nicotinic acetylcholine receptor (nAChR) mediated component of toxicity. Non-oxime bispyridinium compounds (BPDs) have been shown previously to partially counteract the effects of NAs at skeletal muscle tissue, and this has been attributed to inhibition of the muscle $\mathrm{nAChR}$. Functional data indicate that, by increasing the length of the alkyl linker between the pyridinium moieties of BPDs, the antagonistic activity at nAChRs can be improved. Molecular dynamics simulations of the adult muscle nAChR in the presence of BPDs identified key residues likely to be involved in binding. Subsequent two-electrode voltage clamp recordings showed that one of the residues, $\varepsilon Y 131$, acts as an allosteric determinant of BPD binding, and that longer BPDs have a greater stabilizing effect on the orthosteric loop $\mathrm{C}$ than shorter ones. The work reported will inform future design work on novel antidotes for treating NA exposure.
\end{abstract}




\section{Introduction}

Organophosphorus nerve agents (NAs) such as sarin and soman possess a common phosphonate group and irreversibly inactivate the enzyme acetylcholinesterase (AChE) ${ }^{1}$. This occurs by formation of a covalent bond between the NA phosphonate group and the AChE active site serine residue. Inactivation of AChE causes repeated binding of ACh to synaptic receptors without the clearance necessary to terminate the response to nerve impulses. Clinically this manifests itself in a number of ways, including initial fasciculation of muscle tissue mediated by the fast, excitatory response of endplate nicotinic acetylcholine receptors ( $n A C h R s$ ), subsequent paralysis of this tissue due to depolarising block of $\mathrm{nAChRs,},{ }^{2,3}$ and ultimately death. ACh also binds to muscarinic acetylcholine receptors (mAChRs), and other tissue specific symptoms therefore arise, including bronchoconstriction and increased secretions. Expression of nAChRs and mAChRs in the brain leads to centrally-mediated effects ${ }^{4}$ and contributes to apnoea and seizures.

Several small-molecule therapeutics are available to treat NA exposure including atropine, oximes and benzodiazepines. Atropine is a competitive antagonist of mAChRs while benzodiazepines dampen the increased brain excitability that leads to seizures in NA exposed patients. Oxime therapy reactivates AChE through the hydrolysis of the NA-AChE phosphonate bond. At first glance it appears to be a panacea for reversing the enzyme inhibition. However, oxime therapy is limited by ageing of some AChE nerve agent adducts (such as the AChE-soman adduct), which renders reactivation by oximes impossible or too slow to be beneficial. Also, and sometimes additionally, steric hindrance about the AChE-NA adduct prevents nucleophilic attack by oximes, and hence enzyme reactivation ${ }^{5}$. For these reasons, individual oximes are not universal for all NAs, and in an emergency, when initially unable to identify the NA, it would be very difficult to determine the correct oxime to administer sufficiently quickly for optimal therapeutic effect.

Previous experimental work has shown that removing the reactive group of oximes $(-\mathrm{CH}=\mathrm{NOH})$ produces non-oxime bispyridinium compounds (BPDs) that are still able to counteract the muscle effects of NAs ${ }^{5}$. Single channel analysis demonstrated that these non-oxime BPDs inhibit the muscle $\mathrm{nAChR}$ by a negative allosteric mechanism ${ }^{6}$. Negative allosteric modulation of $\mathrm{nAChRs}$ is therefore an effective strategy for counteracting NA toxicity, given the large synaptic ACh concentrations that would otherwise outcompete orthosteric antagonists.

Inhibition of muscle nAChRs by BPDs is influenced by the length of the alkane linker connecting the two pyridinium moieties in these compounds ${ }^{7}$. Interestingly, linkers longer than 6 methylene groups markedly change the slope of the BPD concentration-response curves, suggesting a unique and common binding mechanism for longer chain BPDs. Radioligand binding data also indicate that 
BPDs displace $\left[{ }^{3} \mathrm{H}\right]$ epibatidine from Torpedo Californica nAChRs ${ }^{8}$, suggesting an orthosteric component to the binding. Additionally, the presence of a quaternary charged nitrogen atom is a common pharmacophore of ligands that interact with Cys-loop receptors via cation- $\pi$ interactions ${ }^{9}$. An analogous compound to the BPDs, suberyldicholine dichloride, $\left(\mathrm{CH}_{2}\right)_{6}\left(\mathrm{CO}_{2} \mathrm{CH}_{2} \mathrm{CH}_{2} \mathrm{~N}^{+} \mathrm{Me}_{3}\right)_{2} \cdot 2$ $\mathrm{Cl}^{-}$, interacts bitopically at Torpedo californica nAChRs at both the orthosteric site and a more distant extracellular domain residue $\alpha \mathrm{W} 86{ }^{10}$. Intriguingly, BPDs can act as positive allosteric modulators at human $\alpha 7 \mathrm{nAChRs}{ }^{11}$. It is unclear to what extent the behaviour and mode of action of BPD-like compounds are similar at different nAChR subtypes.

Given this information, we built a homology model of the adult muscle nAChR based on the $\alpha 3 \beta 4$ ganglionic nicotinic receptor ${ }^{12}$ template, docked in two BPDs with 5 (compound MB442) or 8 (compound MB505) methylene groups in the linker region at both binding interfaces, and ran unbiased MD simulations to probe key BPD-nAChR interactions and help explain the changes in Hill slope observed experimentally. From these simulations, we selected amino acid residues that appeared to participate in the potential allosteric component of bitopic BPD interactions and performed site directed mutagenesis (SDM) to alanine of these residues. Two-electrode voltage clamp recordings from Xenopus oocytes containing wild type (WT) or mutant $\alpha \beta \sigma \varepsilon n A C h R s$ showed that $\varepsilon \mathrm{Y} 131$, on loop $\mathrm{E}$ of the complementary face of the $\alpha \varepsilon$ subunit interface, participates as an allosteric component of BPD interaction, and that the equivalent residue, $\delta Y 134$, does not. Observations from the simulations show that MB442 can access two metastable states that confer distinct loop C configurations, whilst MB505 maintains a single stable upward N-terminal facing state. Specific interactions at the $\alpha \varepsilon$ interface over the $\alpha \delta$ interface pave the way to subunit-specific extracellular domain structure-based design of asymmetric BPDs.

\section{Materials and Methods}

\subsection{Homology modelling}

Full length sequences of human adult end-plate $\mathrm{nAChR}$ subunits $(\alpha, \beta, \delta$ and $\varepsilon$ ) were aligned with $\alpha 3$ and $\beta 4$ template sequences and additional phylogenetically related cation channel subunits in $\operatorname{MUSCLE}^{13}$ (SI Fig. 1), before being manually edited to obtain the final alignment. This included removal of $\alpha$ isoform 2 specific residues numbered as 122-146 in SI Fig. 1. The cryo-electron microscopy structure of the $\alpha 3 \beta 4$ ganglionic nicotinic receptor (Protein Data Bank (PDB): 6pv7) ${ }^{12}$ was used as the template for modelling. MODELLER $v 9.12^{14}$ was used to generate a single model first. Additional loop modelling on three regions with non-conserved flexible loops (PDB residues 
562-572, 969-977 and 1768-1776) was performed with 10 models generated for each loop. Loops were selected based on the best Discrete Optimised Protein Score (DOPE) for all conformations, with 31 generated models before final selection ( 1 for initial model and $10 \times 3$ for each loop). Due to non-resolved intracellular domain (ICD) density for the template structure, these residues were excluded from the final model. Residues are numbered following their uniprot sequence and include signal peptide sequences.

\subsection{Docking}

BPDs were docked using a grid box spacing of $1.0 \AA$, with the docking cube centred on the aromatic box. Exhaustiveness was set to 8 and docking calculations performed in Vina 1.1.2 ${ }^{15}$. For all ligands, Gasteiger charges ${ }^{16}$ were added and non-polar $\mathrm{H}$ atoms merged using Autodock 1.5.6 ${ }^{17}$. Final docked poses were selected based on the proximity of a pyridinium ring to the centre of geometry of aromatic box residues $\alpha \mathrm{Y} 113, \alpha \mathrm{W} 169, \alpha \mathrm{Y} 218$ and either $\delta \mathrm{W} 78$ or \&W72.

\subsection{Simulations}

The homology model was embedded in a 100\% 1-palmitoyl-2-oleoyl-sn-glycero-3-phosphocholine (POPC) bilayer using inflategro.pl ${ }^{18}$ before being solvated using a TIP3P water model ${ }^{19}$. Na and $\mathrm{Cl}$ ions were introduced to neutralize the system before additional $\mathrm{Na}$ or $\mathrm{Cl}$ ions were added to make the system up to a final concentration of $0.15 \mathrm{M}$. This system was then energy minimised via the steepest descent algorithm (step size $0.01 \mathrm{~nm}$, max number of steps 50000). Electrostatics were calculated using the Particle Mesh Ewald algorithm ${ }^{20}$. H-bonds where constrained using LINCS ${ }^{21}$ to allow a 2 fs time step. AMBER 99 FFSB-ILDN was used as the protein force field ${ }^{22}$. Each system was equilibrated in the NPT ensemble for $1 \mathrm{~ns}$ using semi-isotropic Berendsen pressure coupling (tau $=1.0)$ and temperature coupling was achieved with $\mathrm{V}$-rescale $(\operatorname{tau}=0.1)$ at $310 \mathrm{~K}$. Backbone atoms were restrained with a $1000 \mathrm{kj} / \mathrm{mol}^{-1} / \AA^{2}$ force constant to allow solvent and lipid molecules to equilibrate around the protein. The resulting system was equilibrated further in the NPT ensemble for 5 ns using semi-isotropic Parrinello-Rahman ${ }^{23}$ pressure coupling (tau $\left.=1.0\right)$ and Nose-Hoover ${ }^{24}$ temperature coupling (tau $=0.5$ ) at $310 \mathrm{~K}$. Holonomic constraints were applied to all-bonds using the LINCS algorithm and position restraints removed to allow proper equilibration of the protein. Production runs were continued in this ensemble. Harmonic restraints were applied to the residues at the base of the extracellular domain to maintain the pentameric structure. These 
regions are remote from the orthosteric site.

\subsection{Molecular Biology}

Human muscle cDNA subunits were provided by Professor David Beeson and subcloned into pcDNA3.1 (Invitrogen). The QuikChange mutagenesis kit (Stratagene, Europe) was used to perform site directed mutagenesis (SDM). Mutated sequences were confirmed by complete sequencing of the cDNA insert (Source Bioscience, Oxford). Residues are numbered with respect to the full-length receptor, including the signal peptide sequence.

\subsection{Expression of nAChRs in Xenopus Oocytes}

Xenopus laevis oocytes were purchased from Xenopus One (Michigan USA). Toad ovary dissection was performed according to UK Home Office regulations. Oocytes were placed in OR2 solution (2.5 mM HEPES (2-[4-(2-hydroxyethyl)piperazin- 1-yl]ethanesulfonic acid), $2 \mathrm{mM} \mathrm{MgCl,} 2 \mathrm{mM} \mathrm{KCl,} 82$ $\mathrm{mM} \mathrm{NaCl}$ ). The $\mathrm{pH}$ of the solution was adjusted manually via titration of $\mathrm{NaOH}$ or $\mathrm{HCl}$ until a $\mathrm{pH}$ of 7.6 was reached. Mature oocytes were isolated and placed in a $2 \mathrm{mg} / \mathrm{ml}$ type IA collagenase OR2 solution on a rotating platform for up to $2 \mathrm{~h}$ at room temperature, to facilitate enzymatic digestion of theca folliculi and epithelial layers. Oocytes were then placed in Barth's medium (1 mM KCl, $88 \mathrm{mM}$ $\left.\mathrm{NaCl}, 2.4 \mathrm{mM} \mathrm{NaHCO}_{3}, 0.3 \mathrm{mM} \mathrm{Ca}\left(\mathrm{NO}_{3}\right)_{2}, 0.82 \mathrm{mM} \mathrm{MgSO}_{4}, 0.41 \mathrm{mM} \mathrm{CaCl}_{2}, 15 \mathrm{mM} \mathrm{HEPES}\right)$ at 18 ${ }^{\circ} \mathrm{C}$ with $1 \mu \mathrm{g} / \mathrm{ml}$ penicillin-streptomycin-amphoterecin B solution (Sigma-Aldrich Ltd., Dorset, UK) and $5 \%$ horse serum at $\mathrm{pH} 7.6$.

Equal ratios of WT or mutant human $\mathrm{nAChR} \alpha 1, \beta 1, \delta$ or $\varepsilon$ subunit $\mathrm{cDNAs}$ were co-injected into oocyte nuclei at a $50.6 \mathrm{nl} /$ oocyte volume using a Nanoject Automatic Oocyte Injector (Drummond, Broomall, PA, USA). 2 ng of total cDNA was injected per oocyte. Oocytes were incubated for 2-5 days at $18{ }^{\circ} \mathrm{C}$ in modified Barth's solution with the previously specified antibiotic/antimycotic mixture post-injection.

Microinjection needles were prepared with glass capillaries (Sartorius, UK) drawn using a horizontal microelectrode puller (Campden Instruments, UK). Electrode tips were manually broken with surgical forceps to provide an approximate 1-1.5 $\mu \mathrm{m}$ wide opening. Needles were filled with mineral oil before cDNA aspiration with a Nanoject II microinjector (Drummond, USA). Oocytes were placed 
individually in wells of a 96-well plate and incubated for up to 5 days at $18^{\circ} \mathrm{C}$.

\subsection{Electrophysiology and concentration response curves}

Oocytes were impaled with two electrodes filled with $3 \mathrm{M} \mathrm{KCl}$ and their membrane potential was maintained at $-60 \mathrm{mV}$ throughout the experiment. All recordings were performed at $18{ }^{\circ} \mathrm{C}$, and cells were perfused with Ringer's solution $\left(\mathrm{KCl} 2.8 \mathrm{mM}, \mathrm{NaCl} 150 \mathrm{mM}, \mathrm{CaCl}_{2} 1.8 \mathrm{mM}\right.$, HEPES $10 \mathrm{mM}$; $\mathrm{pH} 7.4$ adjusted with $\mathrm{NaOH}$ or $\mathrm{HCl}$ ). Currents were recorded using an automated platform equipped with a standard two electrode voltage-clamp configuration (HiClamp; Multi Channel Systems, Reutlignen, Germany). This system differs from standard electrophysiology and other automated platforms because instead of applying the compound in the perfusion, the oocyte is moved into a well from a 96 -well microtiter plate containing $230 \mu \mathrm{l}$ of the desired solution. Data were filtered at 10 $\mathrm{Hz}$, captured at $100 \mathrm{~Hz}$, and analysed using proprietary data acquisition and analysis software running under Matlab (Mathworks Inc., Natick, MA) or Excel (Microsoft, Redmond, WA) combined with GraphPad Version 5 software (CA, USA). Serial dilution of ACh in Ringer's solution was done from frozen stock of $10 \mathrm{mM}$ on the day of the experiments. 6 to 8 point log concentration-response curves (CRCs) were obtained for all constructs. All currents were normalised relative to the maximal WT ACh response (1 mM). Half-log Incremental increases of MB505 concentration were co-applied with an approximate $\mathrm{ACh} \mathrm{EC}_{50}(40 \mathrm{uM})$ concentration to obtain $\mathrm{IC}_{50}$ values. Washes were $3 \mathrm{~min}$ in duration between drug applications. At least two $\mathrm{EC}_{100} \mathrm{ACh}$ applications were performed at the start of each experiment and intermittently after every two drug applications, to eliminate run-up or rundown of responses from being included in subsequent data analyses, and to assess oocyte stability.

\subsection{Data analysis}

All data points were normalised to two $E C_{100}$ control applications before and after the data point concentration applied. All CRCs were fitted with a logistic regression four-parameter Hill equation using Prism 5 software (Graph Pad, CA, USA) (Eq. 1).

$$
Y=\text { Bottom }+\frac{\text { Top-Bottom }}{\left(1+10^{\left(E C_{50}-x\right) * \text { Hillslope }}\right)} \quad \text { Eq. } 1
$$


Top presents the maximal values of each curve whilst Bottom was constrained to zero. $E C_{50}$ represents the concentration of ACh eliciting $50 \%$ of $\mathrm{nAChR}$ maximum response (Imax). HillSlope corresponds to the linear portion of the $I C_{50}$ curve and $x$ is ACh concentration. BPD CRCs were plotted with a four-parameter Hill equation, taking the same form as the above equation, but with $E C_{50}$ replaced by $I C_{50}$ values. This gave the concentration of antagonist (BPD) required to inhibit the receptor response to $50 \%$. Individual $\mathrm{EC}_{50}$ and $\mathrm{IC}_{50}$ values for each experiment were plotted and analysed by one-way analysis of variance (ANOVA) and Dunnett post hoc test. A $P$ value of less than 0.05 was deemed significantly different.

\section{Results}

\subsection{Simulations suggest an allosteric interaction site for BPDs}

To address the question of what causes the changing Hill slope observed by Ring et al. ${ }^{7}$ and to explore interactions of BPDs with the receptor, we constructed a homology model (Materials and Methods Section 2.1) of the human adult muscle $n A C h R$ using the recent $\alpha 3 \beta 4$ ganglionic nicotinic receptor ${ }^{12}$ as a template (Fig. 1A). Prior to docking the BPDs, we assessed the behaviour of this model with ACh (Table 1), to help assess the quality of the model for reproducing known modes of interaction for this endogenous ligand. In these simulations, ACh bound in stable manner, remaining in the aromatic box, with no unbinding events observed in all 10 repeated simulations (Fig. 1B-D). Although a long rightward skew can be observed for the $\alpha \delta$ interface $A C h$, root mean square deviation (RMSD) analysis (Fig. 1B - blue histogram) of individual trajectories demonstrated that, for simulations where RMSDs appear large or skewed, the final binding mode of ACh resided within the aromatic box. 


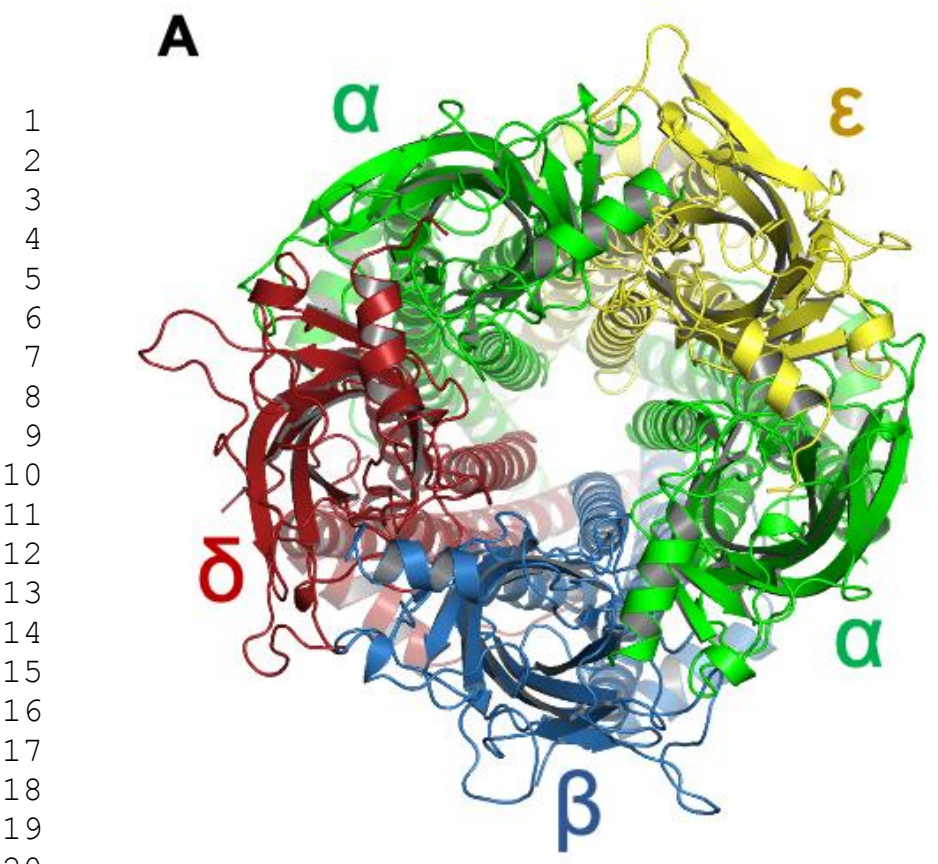

B
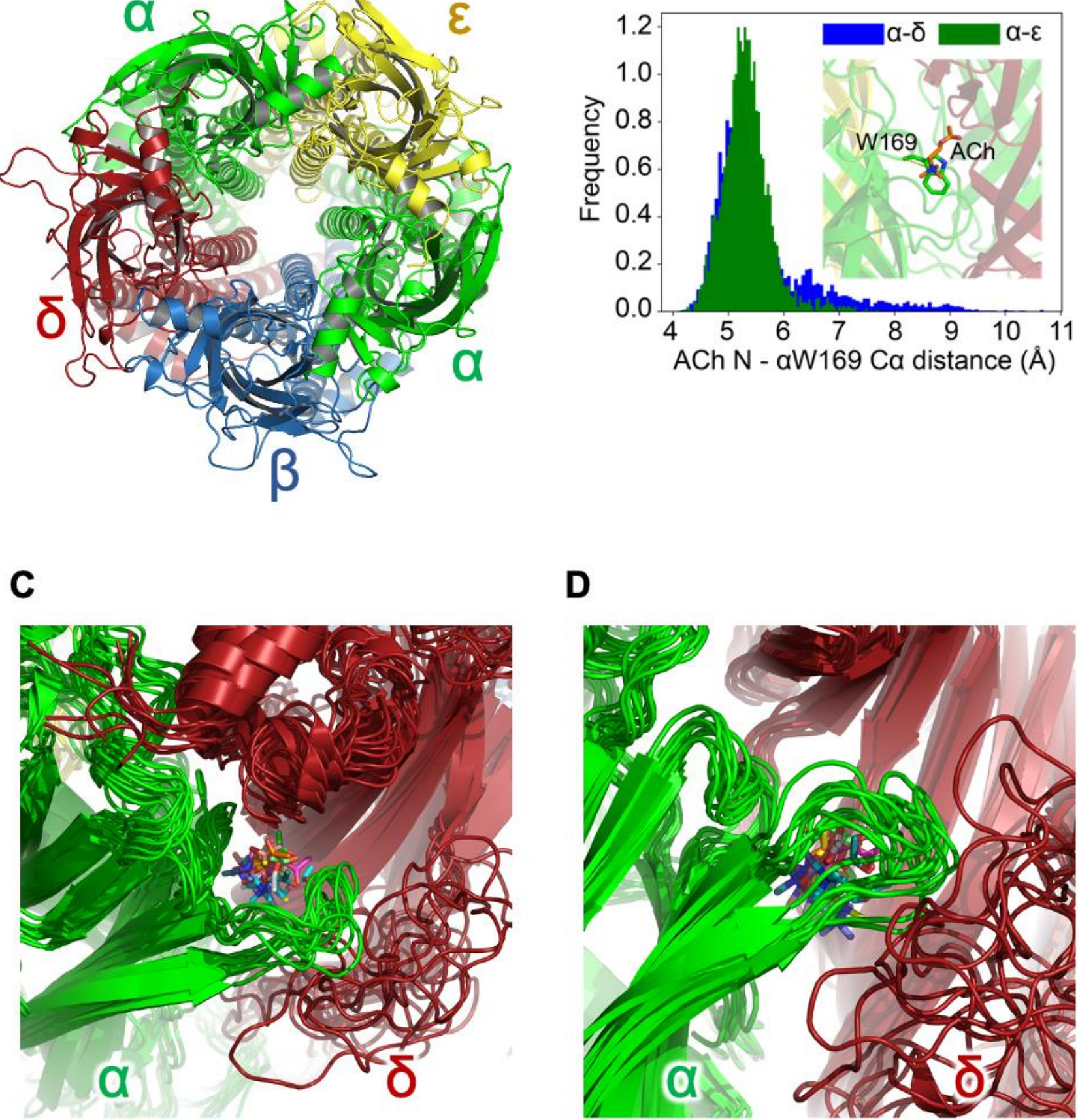

D

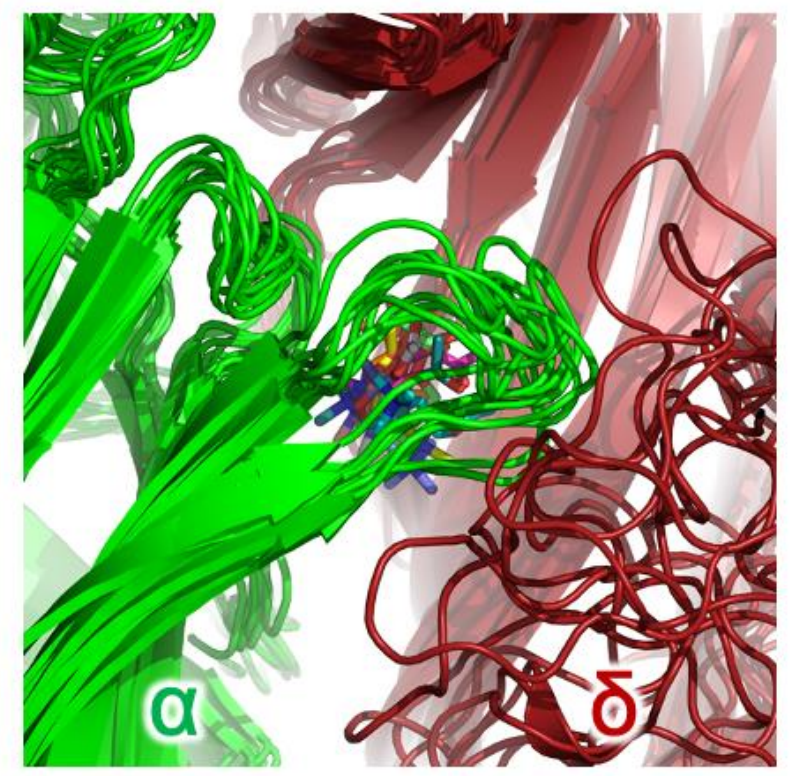

Figure 1. (A) Model of the human $n A C h R$ viewed top down showing a (green), $\beta$ (blue), $\delta$ (red) and $\varepsilon$ (yellow) subunits. (B) shows the distribution of the distance between the ACh quaternary nitrogen atom and $\alpha W 169 \mathrm{C} \alpha$ atom, with 5000 frames over $1 \mu \mathrm{S}$ of aggregate simulation time used to obtain the histograms. The insert shows a representative binding mode with ACh depicted in orange stick form and $\alpha W 169$ in green stick form. Oxygen and nitrogen atoms are coloured red and blue respectively. The dashed yellow line shows the collective variable under analysis. As the $\alpha \delta$ interface possesses a large skew, we analysed each individual simulation to check for unbinding events. (C) and (D) show two perspectives of the last frames from each of the 10 simulation replicas at the $\alpha \delta$ subunit interface superimposed. For all simulations, ACh remained bound to the orthosteric site. 
We proceeded to assess the stability and binding modes of the BPD compounds MB442 and MB505 (with 5 or 8 methylene groups in their linkers, respectively) within the orthosteric binding site. These compounds were chosen as their Hill slopes are located at the extremes of changing Hill Slope values as a function of alkyl linker length ${ }^{7}$, allowing useful comparison of potential changing mechanisms between the two molecules. MB442 and MB505 were individually docked at both binding interfaces (Materials and Methods Section 2.2). Poses were selected based on the lowest distance between a BPD nitrogen atom and the same key aromatic box residues used for ACh docking. The binding mode of the non-orthosteric BPD nitrogen atom was not considered for pose selection. Each docked solution was subjected to 10 repeated 100 ns unbiased MD simulations (Table 1). Across all BPDs, and as expected for a molecule with a quaternary nitrogen atom ${ }^{25}$, one end of the molecule remained stable within the aromatic box (Fig. 2) of the orthosteric binding site. Peak densities of the nitrogen to backbone Ca of $\alpha \mathrm{W} 169$ (as calculated for ACh, Fig. 1B) were observed at around 5-6 Å for all compounds studied (Fig. 2).

Table 1. Summary of simulations

\begin{tabular}{ccc}
\hline & $\begin{array}{c}\alpha-\delta \text { interface } \\
n \text { (total time in } n s)\end{array}$ & $\begin{array}{c}\alpha-\varepsilon \text { interface } \\
n \text { (total time in ns) }\end{array}$ \\
\hline ACh & $10(1000)$ & $10(1000)$ \\
MB442 $(\mathrm{n}=5)$ & $10(1000)$ & $10(1000)$ \\
MB505 $(\mathrm{n}=8)$ & $10(1000)$ & $10(1000)$
\end{tabular}

The letter $\mathrm{n}$ refers to the number of alkylene groups $\left(\mathrm{CH}_{2}\right)_{n}$ separating the two pyridinium units of the BDPs (see Fig. 2).

Both orthosteric components of the BDPs remained stable within the aromatic box of each interface as measured by the distance between the $\alpha \mathrm{W} 169 \mathrm{C} \alpha$ atom and BPD nitrogen atom of the pyridinium ring closest to aW169 (Fig. 2). Peak densities occurred between 5 and $6 \AA$ for both MB442 and MB505 with no appreciable difference between ligands or interfaces. 
A

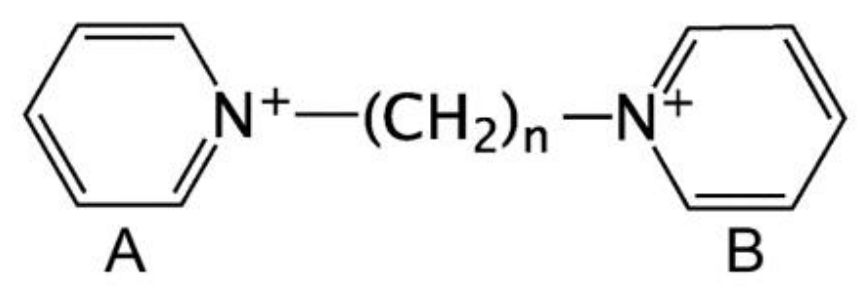

MB442: $n=5$. MB505: $n=8$.

B
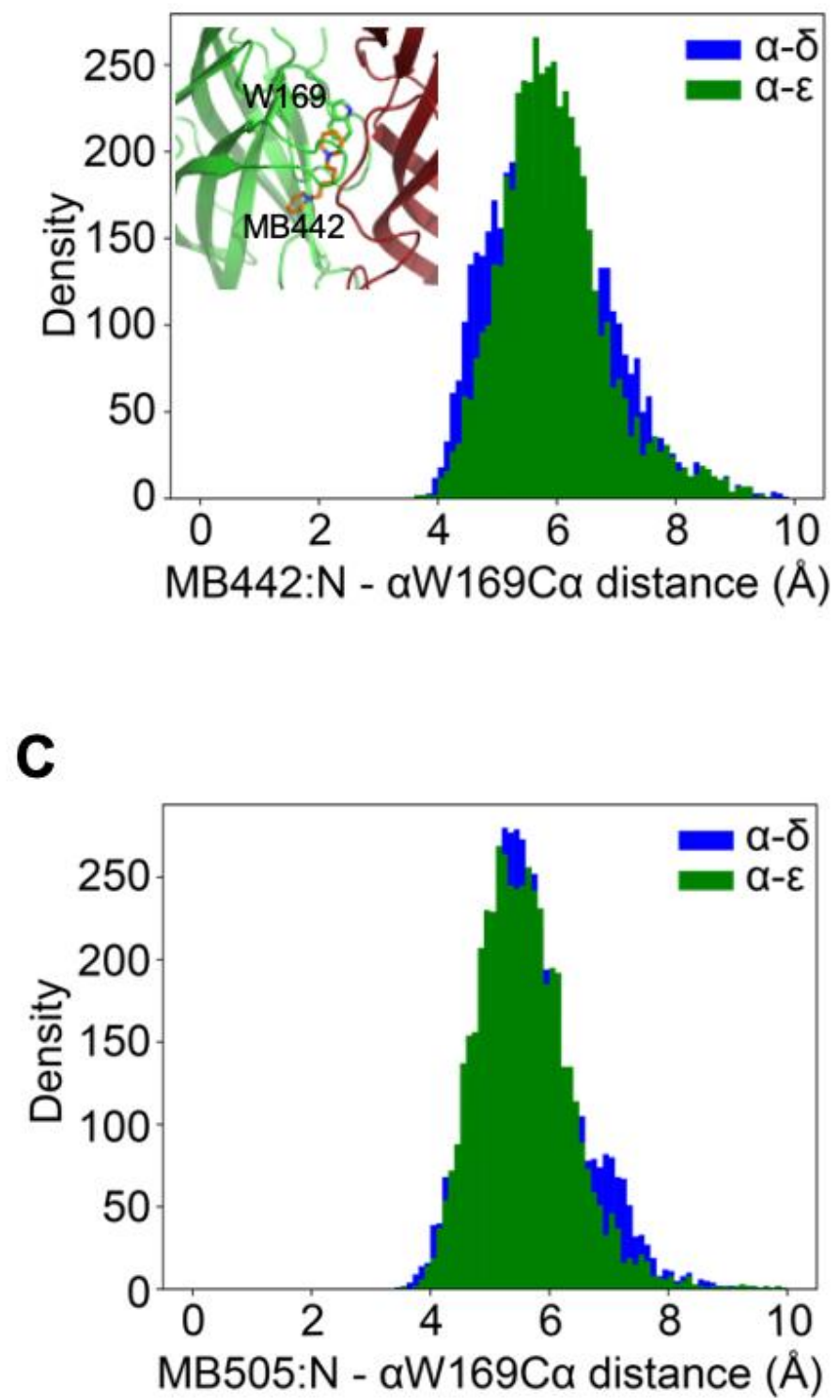

Figure 2. (A) Structures of MB442 and MB505. (B) Density plots of the distance between the $B P D$ pyridinium nitrogen atom and centre of geometry of aW169 at both orthosteric interfaces for MB442 and (C) MB505. The insert in (B) is a representative snapshot of MB442 (orange) in the down state at the $\alpha-\delta$ interface with $\alpha W 169$ shown in a green stick representation. 
For compounds that act at the orthosteric binding site, distinct loop $\mathrm{C}$ conformations are known to be associated with either agonist or antagonist binding modes. While the ability of agonists to elicit their effects is associated with loop C closure, antagonists are thought to prevent receptor activation by restricting loop $C$ conformations to a more outward conformation ${ }^{26,27}$. From initial analysis of individual trajectories, two distinct BPD conformations could be observed for MB442 $(n=5)$. We therefore analysed the binding mode of each BPD with respect to the distance between the centre of geometry of loop $\mathrm{C}$ disulfide sulfur residues and the $\alpha W 169-C \alpha$ atom (Fig. 3). The more closed loop C conformation, around $11 \AA$ (Fig. 3A), was associated with a 'down pose' where the allosteric pyridinium moiety was located towards the membrane. A secondary peak was observed at $\sim 14-15 \AA$ which is only accessible to poses we refer to as the "up pose" (where one pyridinium interacts with the orthosteric binding site, while the other locates towards the N-terminal part of the receptor). The larger MB505 $(n=8)$ was incapable of accessing the down-state conformation, with average loop C receptor configurations of around $13 \AA$ (Fig. 3B). 
A

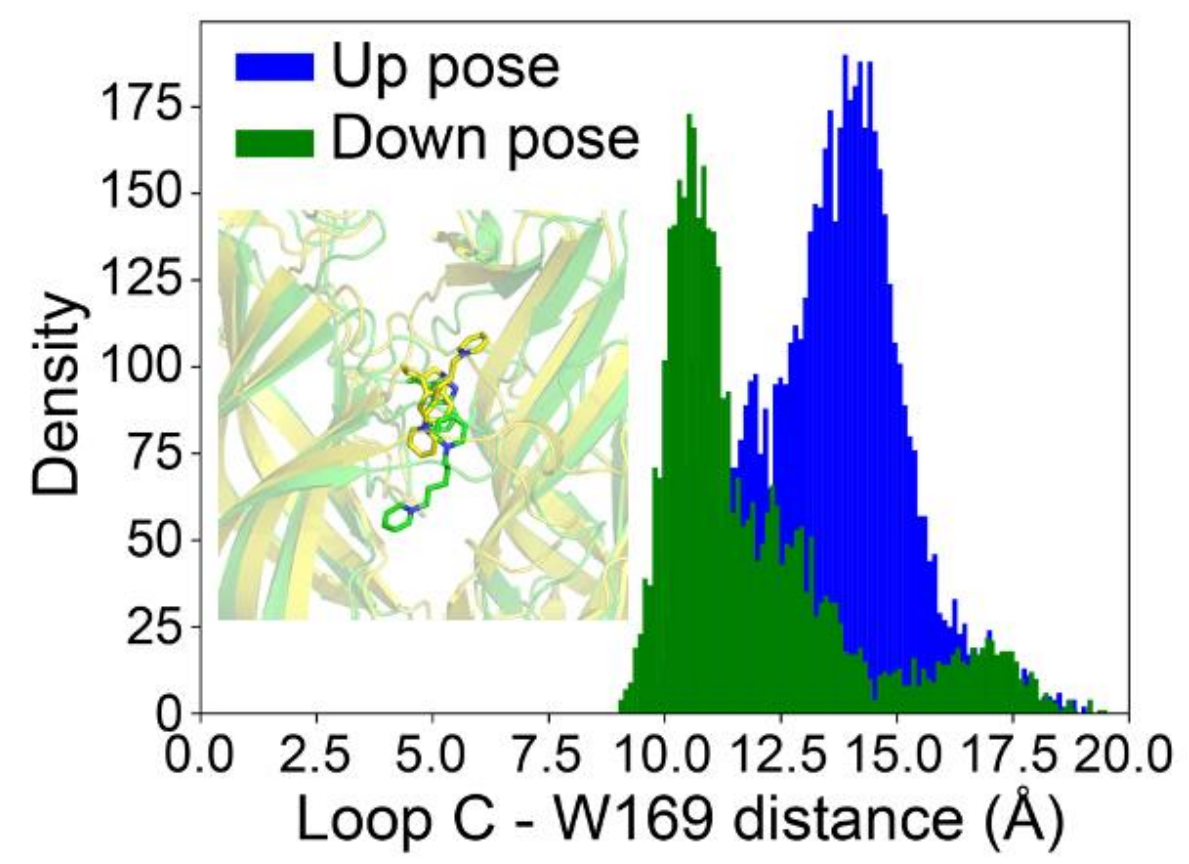

B

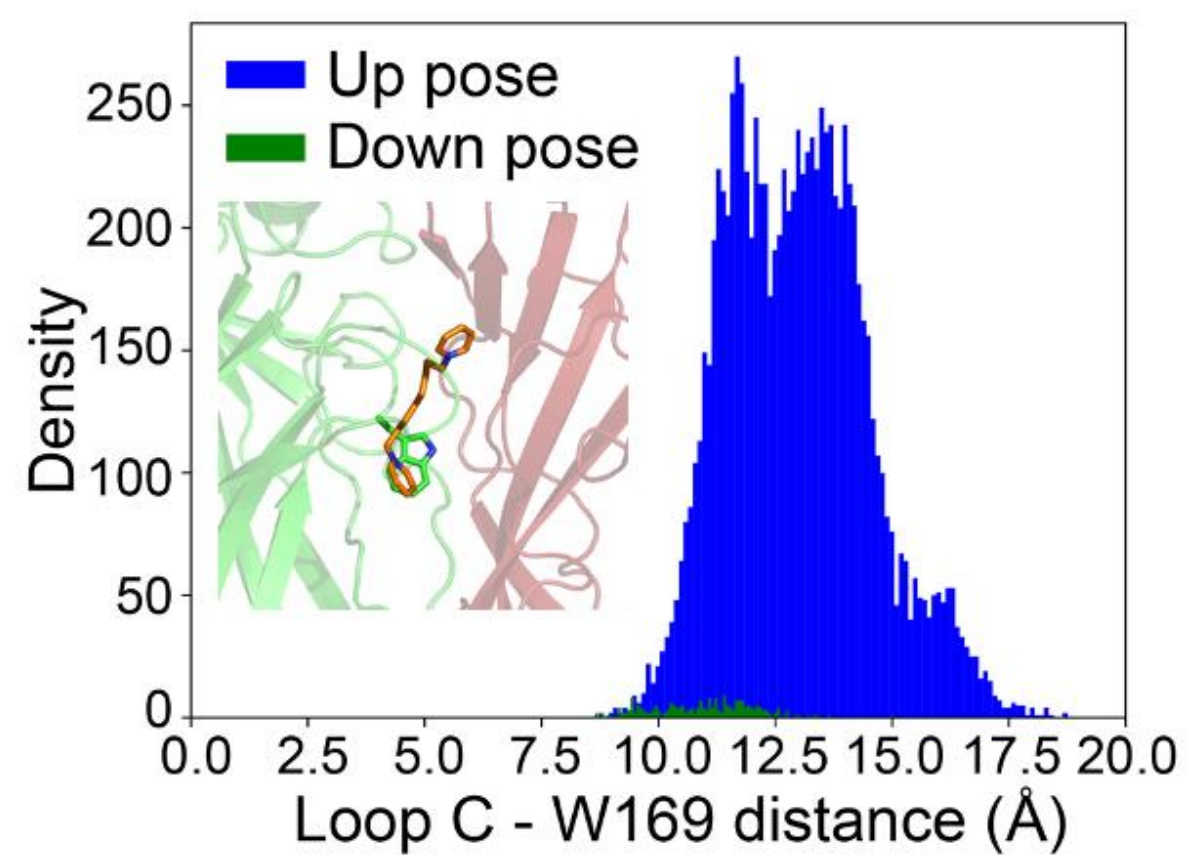

Figure 3. (A) Density plot for centre of geometry of loop $C$ disulfide sulfur atoms from the backbone carbonyl of a conserved aW169 residue as a function of MB442 $(n=5)$ binding pose. The insert shows representative snapshots of 'up' or 'down' poses in green or yellow respectively. (B) Density plot for centre of geometry of loop C disulfide sulfur atoms from the backbone carbonyl of the conserved aW169 residue as a function of MB505 $(n=8)$ binding pose. The insert shows a representative snapshot of MB505 in the 'up' configuration in an orange stick representation. 
Given that antagonist activity is greatest for longer chain BPDs ${ }^{7}$, we analysed the up-pose to determine if this conformation might be responsible for the pharmacological effects outlined

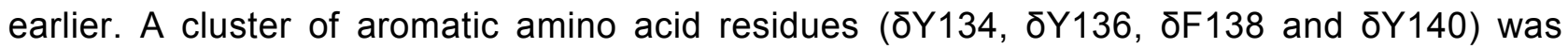
identified on the $\delta(-)$ complementary orthosteric face within plausible reach of the allosteric pyridinium moiety (Fig. 4A). Additionally, a single conserved tyrosine residue at $\varepsilon$ Y131 was also identified. We calculated, for each independent simulation, the percentage of time when a pyridinium nitrogen atom was within $7 \AA$ of the centre of geometry of $\delta Y 134, \delta Y 136, \delta F 138$ and $\delta Y 140$, or also within $7 \AA$ of $\varepsilon Y 131.7 \AA$ was chosen as the cut-off value to account for all possible geometries of aromatic-aromatic interactions.

Notable differences between both ligands and interfaces were observed, with increased percentage contact for MB505 found at both interfaces compared to MB442. Despite this, the MB505 interactions at the $\alpha-\delta$ interface were substantially lower compared to the $\alpha-\varepsilon$ interface, with percentage contacts for $\varepsilon Y 131$ occurring over $63 \%$ of the time at a minimum for all simulations, and over $90 \%$ of the time for 7 out of 10 of the independent simulations (Fig. 4). Given that $\varepsilon Y 131$ is conserved at the $\delta$ interface (סY134), we analysed percentage contacts again at the $\alpha-\delta$ interface, focusing exclusively on $\delta Y 134$. While such contact values were increased with respect to the aggregated $\delta$ residue analysis, they were still lower than $\varepsilon Y 131$ interactions overall, with 5 out of 10 simulations falling below $60 \%$ contact and only one simulation attaining over $90 \%$. For MB442, $7 / 10$ simulations fell below $10 \%$, with one single simulation reaching 59\% contact (SI Fig. 2). 
A

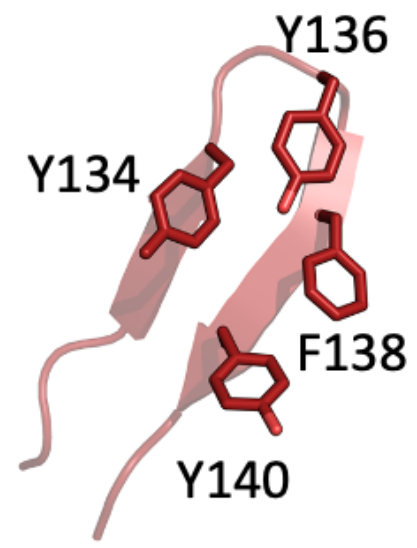

C

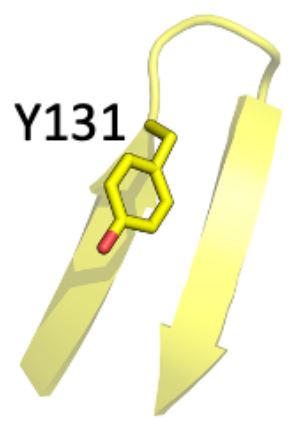

B

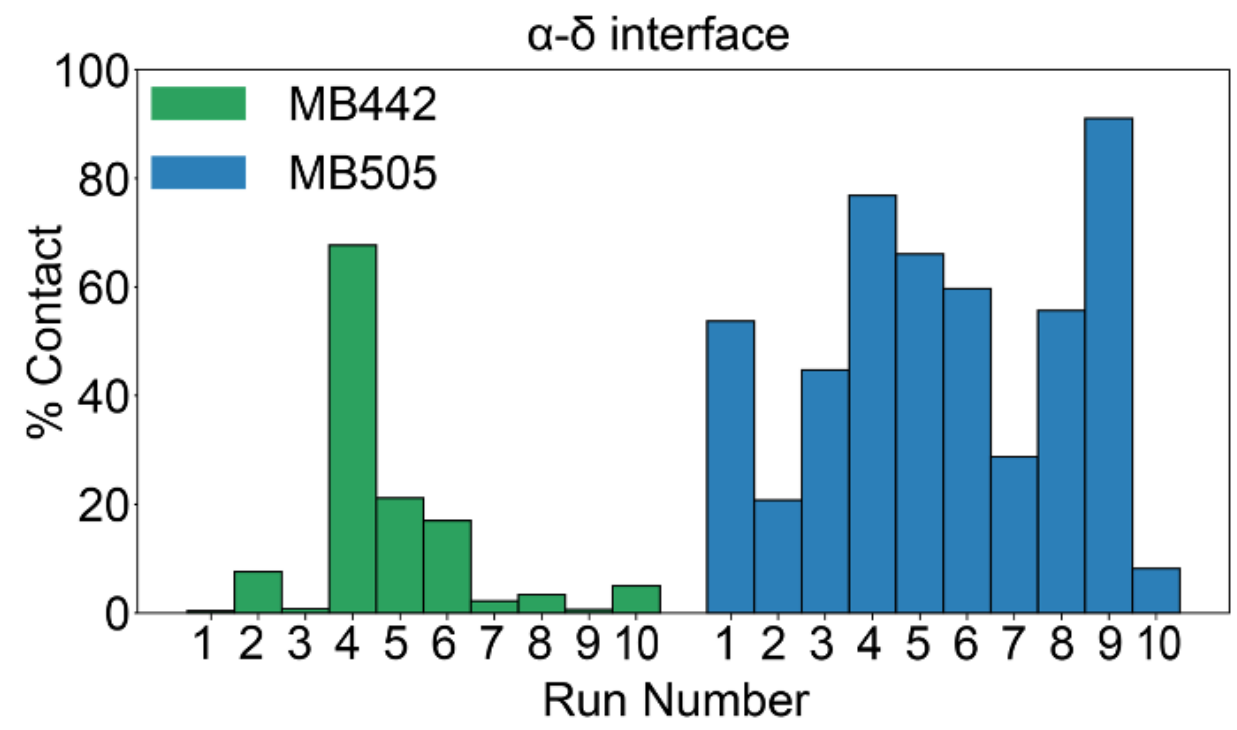

D

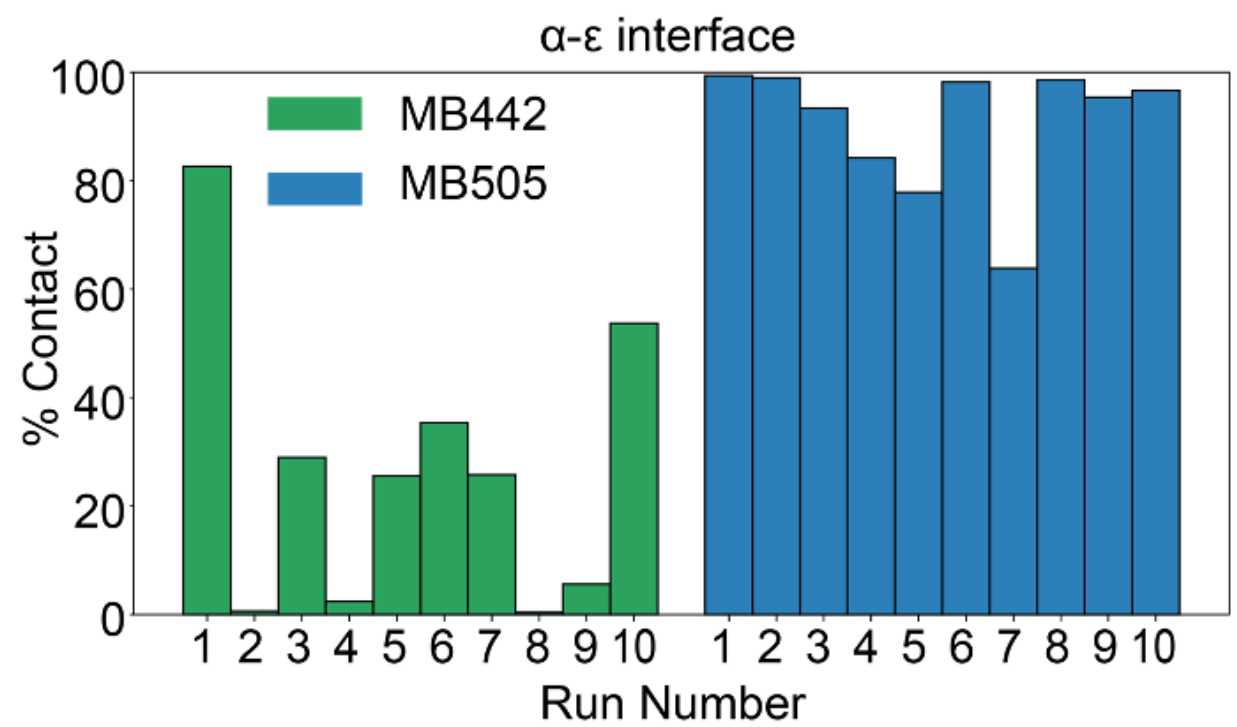

Figure 4. (A) shows the aromatic cluster on the $\delta(-)$ face. (B) shows $\%$ of each independent simulation where a pyridinium nitrogen atom falls within $7 \AA$ of the centre of geometry of residues $\delta Y 134, \delta 136$ and $\delta F 138$, with MB442 represented as green bars and MB505 as blue bars. (C) shows $\varepsilon Y 131$ on the $\varepsilon(-)$ face. (D) shows \% of each independent simulation where a pyridinium nitrogen falls within $7 \AA$ of the centre of geometry of residue $\varepsilon 131$, with MB442 depicted as green bars and MB505 as blue bars.

\subsection{TVEC Experiments Confirm an Allosteric Interaction}

Using the results from the MD contact analysis to focus subsequent experimental efforts, we performed SDM on residues $\delta \mathrm{Y} 134$ and $\varepsilon$ Y131, mutating them to alanine. The response to increasing ACh concentrations was measured to determine if these mutations conferred any structural importance to the receptor. The observed concentration response curves (CRCs) showed 
no statistically significant change in $\mathrm{EC}_{50}$ between WT and mutant receptors (Fig. 5A and B).

CRCs for MB505 in the presence of $40 \mu \mathrm{M}$ ACh $\left(\mathrm{EC}_{50}\right.$ of $\mathrm{ACh}$ ) were then plotted to derive $\mathrm{IC}_{50}$ values for each construct (Fig $5 \mathbf{C}$ and D, Table 2). The $\varepsilon Y 131 \mathrm{~A}$ mutant produced a 3.42 fold decrease in the inhibitory potency of MB505 (WT receptor $\mathrm{IC}_{50}$, log -3.86; IC $\mathrm{C}_{50}$ mutant receptor, $\log$ $\left.\mathrm{IC}_{50}-3.26 \mathrm{M} ; \mathrm{p}<0.05\right)($ Table 2).

Table 2. Summary of TEVC mutation data

\begin{tabular}{cc}
\hline Construct & $\log \mathrm{IC}_{50}(\mathrm{M})$ \\
\hline WT & -3.86 \\
סY134A & -3.91 \\
$\varepsilon Y 131 \mathrm{~A}$ & -3.33 \\
\hline
\end{tabular}


A

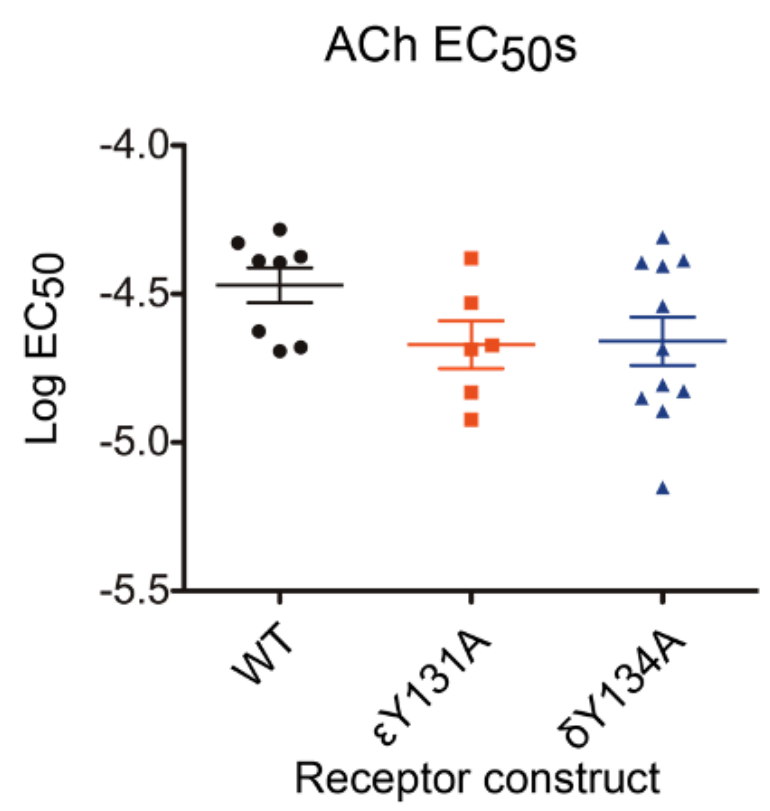

C

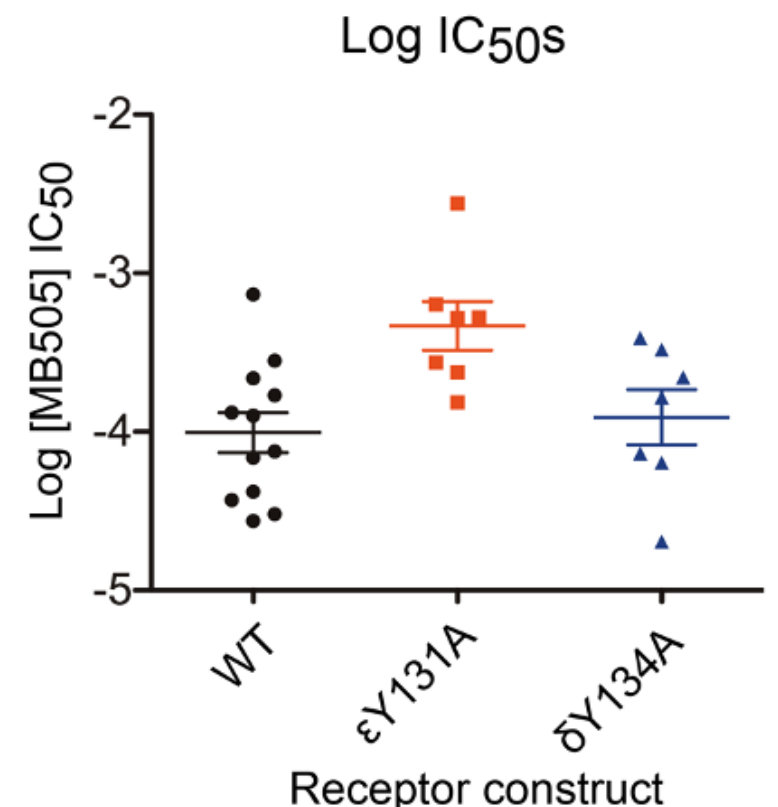

B

CRC for ACh at WT \& mutant muscle nAChRs

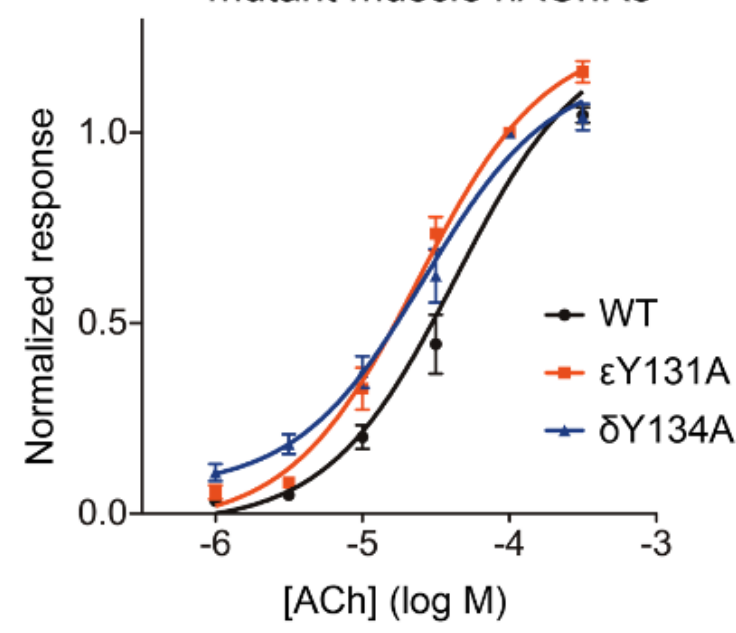

D

MB505 CRC in the presence of $\mathrm{EC}_{50}[\mathrm{ACh}]$

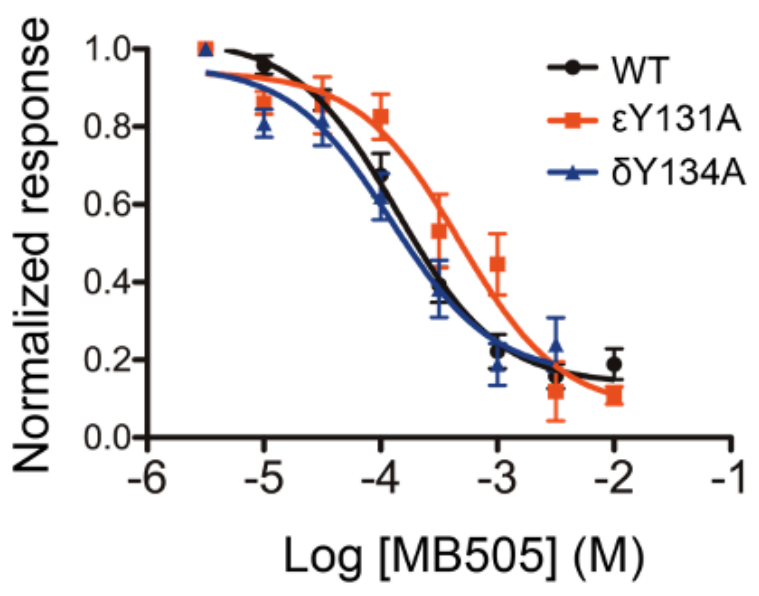

Figure 5. (A) Spread of $E C_{50}$ values from ACh concentration response curves. Mean with SEM. (B) Concentration response curve of ACh at mutant and wild type nAChRs. (C) Spread of $I C_{50}$ values from MB505 concentration response curves. Mean with SEM. (D) Concentration response curve of MB505 in the presence of $\sim E C_{50}[A C h]$ at mutant and wild type nAChRs. 


\section{Discussion}

We sought to elucidate the key molecular determinants of non-oxime BPD interactions at the adult human muscle nAChR. While BPDs have been shown to interact with neuronal subtypes of nAChRs, we focused our attention specifically on adult end-plate receptors, as (i) the blood-brain barrier may preclude certain charged nitrogenous compounds from reaching neuronal receptors in sufficiently high concentrations, and (ii) sigmoidal concentration response curves can be fitted for all BPDs interacting with neuronal receptors, whereas end-plate CRCs show changing Hill slope with different linker lengths ${ }^{7}$. Furthermore, skeletal muscle effects are known to contribute significantly to nerve agent lethality ${ }^{28-31}$ by depressing diaphragm function.

Using MD simulations as a guide to discern potential binding site residues for non-oxime BPDs, we identified residues on the complementary face of the subunits interface as likely candidates. First, we obtained $\mathrm{EC}_{50}$ values for $\mathrm{ACh}$ at WT and mutant receptors. The lack of change between these constructs indicates that these amino acid residues do not affect ACh binding or nAChR gating isomerisation and are genuinely allosteric sites. Interestingly, of the two equivalent tyrosine residues on either $\varepsilon$ and $\delta$ complementary faces ( $\delta \mathrm{Y} 134$ and $\varepsilon \mathrm{Y} 131$ ), only the $\varepsilon$ face was determined to affect nAChR-BPD (MB505) sensitivity. The differences between amino acid composition at both faces are pronounced, with a total of 4 aromatic residues at the $\delta$ face compared to just 1 at the $\varepsilon$ face. It is conceivable that these local changes in amino acid composition could preferentially effect the orientation and mobility of a neighbouring residue, such that conserved amino acids on different faces of the receptor may behave differently.

The presence of a specific molecular determinant to BPD binding at a single subunit interface does provide some explanation to the partial agonist profile of these compounds observed herein and in previous work ${ }^{7}$. Indeed, the preservation of a single subunit capable of binding ACh could still allow for isomerisation of the $\mathrm{nAChR}$ to an active conformation, just with a slower rate of transition 
32,33. Furthermore, the nature of this allosteric interaction is mirrored by a series of compounds known to act in an allosteric manner at the $\alpha 4 \beta 2$ receptor, where residues on the $\beta 2$ subunit in a similar location to those observed here on the $\delta$ and $\varepsilon$ subunits dramatically effect allosteric modulation ${ }^{34,35}$. It seems likely that this location is a target for allosteric modulation across nAChRs. The role of different interfaces and the influence of different subunits is an ongoing area of investigation ${ }^{36}$.

While the presence of a specific molecular determinant might provide an explanation for partial agonist activity, the small observed change in $\mathrm{EC}_{50}$ is unlikely to fully account for the large changes between BPDs as determined by Ring et $a^{7}{ }^{7}$. Analysis of the MD simulations also suggested that distinct loop $\mathrm{C}$ conformations were associated with specific BPD binding modes and that longer length BPDs preferentially adopted the up-state configuration. The similarity between loop C conformations for up-states of both penta- and octa-methylene linked BPDs indicates that antagonist responses as a function of alkyl linker length grade through their ability to access either up or down state configurations and that loop C - aromatic box distances do not change incrementally with the linker length.

These findings support the view that it may be possible to pursue modification of these compounds in an asymmetric fashion, as reported previously, ${ }^{37} 11$ and also to rationally design a compound that is not only nAChR subtype specific, but also interface specific. At increasing lengths, nonoxime BPDs would more optimally bridge both peripheral and catalytic anionic sites of AChE simultaneously, reflected by the increased $\mathrm{plC}_{50}$ values for $\mathrm{AChE}$ relative to $\mathrm{pK}_{\mathrm{i}}$ values for Torpedo californica nAChRs ${ }^{8}$ and in agreement with the proposed binding mode of homologous bitopic symmetrical quaternary ammonium compounds. ${ }^{38}$ Therefore, despite the improved nAChR antagonist profile and allosteric interactions for longer chain BPDs, concurrent increases in AChE affinity may offset the therapeutic efficacy of longer chain BPDs. As such, the relative affinities of long chain non-oxime phenylalkyl pyridinium compounds could be explored as a strategy to improve relative selectivity for $\mathrm{nAChRs}$ over AChE. Additionally, previously determined $\mathrm{pK}_{\mathrm{i}}$ values of both short and long length non-oxime BPDs at Torpedo californica span the low to moderate $m M$ level between $4.7 \pm 0.1 \mathrm{mM}$ for BPDs with 3 methylene groups and up 
to $5.7 \pm 0.0 \mathrm{mM}$ for those with 10 methylene groups. As such, this limits BPD therapeutic applications as well as because of their partial reliance on binding to the orthosteric site.

\section{Conclusion}

Here we provide a mechanistic link for previously described structure-activity relationship data, demonstrating that non-oxime BDPs of longer linker length stabilise loop $C$ of the adult human muscle nAChR upon binding. We find that MB505 (alkylene linker, $n=8$ ) is capable of interacting with $\varepsilon \mathrm{Y} 131$, indicating a possible preference for $\alpha \varepsilon$ over $\alpha \delta$ subunit interfaces. This provides an explanation for non-oxime BPD partial agonism and for the location of an allosteric site that might be exploited in the development of improved nAChR modulators to treat organophosphorus nerve agent exposure.

\section{Acknowledgements}

M.E. is supported by an Engineering and Physical Sciences Research Council (EPSRC) Industrial Cooperative Awards in Science \& Engineering (iCASE) studentship (voucher number 15220076) with DSTL. PCB thanks the BBSRC for funding (BB/S001247/1). This project made use of computation time on JADE (EP/P020275/1) via HECBioSim (http://www.hecbiosim.ac.uk), supported by EPSRC (grant no. EP/R029407/1). We thank Advanced Research Computing (Oxford) for additional computer time.

\section{Author contributions}

P.C.B, T.J.P, A.C.G, C.M.T, J.E.H.T and M.E. conceived the study. M.B. developed synthetic routes to the BPDs. M.E planned the simulation protocol, performed and analyzed the MD simulations. K.B performed additional simulations. M.E. performed and analyzed the electrophysiology and I.B. supervised it. M.E., K.B. and P.C.B analyzed the data. P.C.B., T.J.P and M.E. interpreted the results. P.C.B and M.E. wrote the paper.

\section{Compliance with ethical standards}


Dissection of Xenopus laevis was performed according to UK Home Office regulations.

\section{Conflict of interest}

The authors declare no competing interests.

\section{References}

1 Costanzi, S., Machado, J.-H. \& Mitchell, M. Nerve agents: What they are, how they work, how to counter them. ACS Chem. Neurosci. 9, 873-885, (2018).

2 Cookson, J. C. \& Paton, W. D. M. Mechanisms of neuromuscular block. Anaesthesia 24, 395 - 416, (1969).

3 Paton, W. D. M. \& Waud, D. R. The margin of safety of neuromuscular transmission. J. Physiol. 191, 59-90, (1967).

$4 \quad$ Harrison, P. K., Sheridan, R. D., Green, A. C., Scott, I. R. \& Tattersall, J. E. H. A guinea pig hippocampal slice model of organophosphate-induced seizure activity. J. Pharmacol. Exp. Ther. 310, 678, (2004).

5 Timperley, C. M. et al. 1,1'-(Propane-1,3-diyl)bis(4-tert-butylpyridinium) di(methanesulfonate) protects guinea pigs from soman poisoning when used as part of a combined therapy. MedChemComm 3, 352-356, (2012).

6 Tattersall, J. E. H. lon channel blockade by oximes and recovery of diaphragm muscle from soman poisoning in vitro. Br. J. Pharmacol. 108, 1006-1015, (1993).

7 Ring, A. et al. Bispyridinium compounds inhibit both muscle and neuronal nicotinic acetylcholine receptors in human cell lines. PLOS ONE 10, e0135811, (2015).

8 Niessen, K. V. et al. Affinities of bispyridinium non-oxime compounds to [3H]epibatidine binding sites of Torpedo californica nicotinic acetylcholine receptors depend on linker length. Chem. Biol. Interact. 206, 545-554, (2013).

9 Lynagh, T. \& Pless, S. A. Principles of agonist recognition in Cys-loop receptors. Front. Physiol. 5, 160-160, (2014).

10 Kapur, A., Davies, M., Dryden, W. F. \& Dunn, S. M. J. Tryptophan 86 of the $\alpha$ subunit in the Torpedo nicotinic acetylcholine receptor is important for channel activation by the bisquaternary ligand suberyldicholine. Biochemistry 45, 10337-10343, (2006).

11 Scheffel, C. et al. Electrophysiological investigation of the effect of structurally different bispyridinium non-oxime compounds on human $\alpha 7$-nicotinic acetylcholine receptor activity-An in vitro structure-activity analysis. Toxicology Letters 293, 157-166, (2018).

12 Gharpure, A. et al. Agonist selectivity and ion permeation in the $\alpha 3 \beta 4$ ganglionic nicotinic receptor. Neuron 104, 501-511.e506, (2019).

13 Edgar, R. C. MUSCLE: Multiple sequence alignment with high accuracy and high throughput. Nucleic Acids Research 32, 1792-1797, (2004).

14 Webb, B. \& Sali, A. Comparative protein structure modeling using modeller. Curr. Prot. Bioinf. 5, 5.61-65.66.32, (2014).

15 Trott, O. \& Olson, A. J. AutoDock Vina: improving the speed and accuracy of docking with a new scoring function, efficient optimization, and multithreading. J. Comput. Chem. 31, 455-461, (2010).

16 Gasteiger, J. \& Marsili, M. New model for calculating atomic charges in molecules. Tetrahedron Lett 19, 3181-3184, (1978).

17 Morris, G. M. et al. AutoDock 4 and AutoDockTools 4: Automated docking with selective receptor flexibility. J. Comp. Chem. 30, 2785-2791, (2009).

18 Kandt, C., Ash, W. L. \& Peter Tieleman, D. Setting up and running molecular dynamics simulations of membrane proteins. Methods 41, 475-488, (2007). 
Jorgensen, W. L., Chandresekhar, J., Madura, J. D., Impey, R. W. \& Klein, M. L. Comparison of simple potential functions for simulating liquid water. J. Chem. Phys. 79, 926-935, (1983).

Darden, T., York, D. \& Pedersen, L. Particle mesh Ewald - an N.log(N) method for Ewald sums in large systems. J. Chem. Phys. 98, 10089-10092, (1993).

21 Hess, B. P-lincs: A parallel linear constraint solver for molecular simulation. J. Chem. Theor. Comput. 4, 116-122, (2008).

22 Lindorff-Larsen, K. et al. Improved side-chain torsion potentials for the Amber ff99SB protein force field. Proteins: Struc. Func. Genet. 78, 1950-1958, (2010).

23 Parinello, M. \& Rahman, A. Polymorphic transitions in single crystals - a new molecular dynamics method. J. Appl. Phys. 52, 7182-7190, (1981).

24 Nose, S. A molecular dynamics method for simulations in the canonical ensemble. Mol. Phys. 52, 255-268, (1984).

25 Dougherty, D. A. Cation-p interactions in chemistry and biology: a new view of benzene, phe, tyr and trp. Science 271, 163-168, (1996).

$26 \mathrm{Ma}, \mathrm{Q} .$, Tae, H.-S., Wu, G., Jiang, T. \& Yu, R. Exploring the relationship between nicotinic acetylcholine receptor ligand size, efficiency, efficacy, and c-loop opening. J. Chem. Inf. Model, (2017).

27 Taly, A. et al. Discrimination of agonists versus antagonists of nicotinic ligands based on docking onto AChBP structures. J. Mol. Graph. Model. 30, 100-109, (2011).

Tattersall, J. in Chemical Warfare Toxicology Vol. 2 (ed Royal Society of Chemistry) Ch. 3, (2106). Timperley, C., M. \& Tattersall, J. in Best Synthetic Methods (ed Christopher M. Timperley) 1-89 (Academic Press, 2015). Tattersall, J. E. H. Anticholinesterase toxicity. Curr. Opin. Physiol. 4, 49-56, (2018).

Blaber, L. C. \& Bowman, W. C. Studies on the repetitive discharges evoked in motor nerve and skeletal muscle after injection of anticholinesterase drugs. Br. J. Pharmacol. Chemother. 20, 326344, (1963).

32 Gupta, S., Chakraborty, S., Vij, R. \& Auerbach, A. A mechanism for acetylcholine receptor gating based on structure, coupling, phi, and flip. J. Gen. Physiol., (2016).

33 Purohit, P. \& Auerbach, A. Loop C and the mechanism of acetylcholine receptor-channel gating. J. Gen. Physiol. 141, 467-478, (2013).

34 Henderson, B. J. et al. Negative allosteric modulators that target human $\alpha 4 \beta 2$ neuronal nicotinic receptors. J. Pharmacol. Exp. Ther. 334, 761, (2010).

35 Henderson, B. J. et al. Defining the putative inhibitory site for a selective negative allosteric modulator of human $\alpha 4 \beta 2$ neuronal nicotinic receptors. ACS chemical neuroscience 3, 682-692, (2012).

36 Alcaino, C. et al. Role of the cys loop and transmembrane domain in the allosteric modulation of $\alpha 4 \beta 2$ nicotinic acetylcholine receptors. J. Biol. Chem. 292, 551-562, (2017).

37 Rappenglück, S. et al. Synthesis of a series of non-symmetric bispyridinium and related compounds and their affinity characterization at the nicotinic acetylcholine receptor. ChemMedChem 13, 26532663, (2018).

38 Harel, M. et al. Quaternary ligand binding to aromatic residues in the active-site gorge of acetylcholinesterase. Proc. Natl. Acad. Sci. USA 90, 9031, (1993). 
Click here to access/download Supplementary Material BPD_paper_v14-R1-highlight.docx 
Click here to access/download Supplementary Material Supplementary Information.pdf 


\section{Declaration of interests}

$\bigotimes$ The authors declare that they have no known competing financial interests or personal relationships that could have appeared to influence the work reported in this paper.

$\square$ The authors declare the following financial interests/personal relationships which may be considered as potential competing interests:

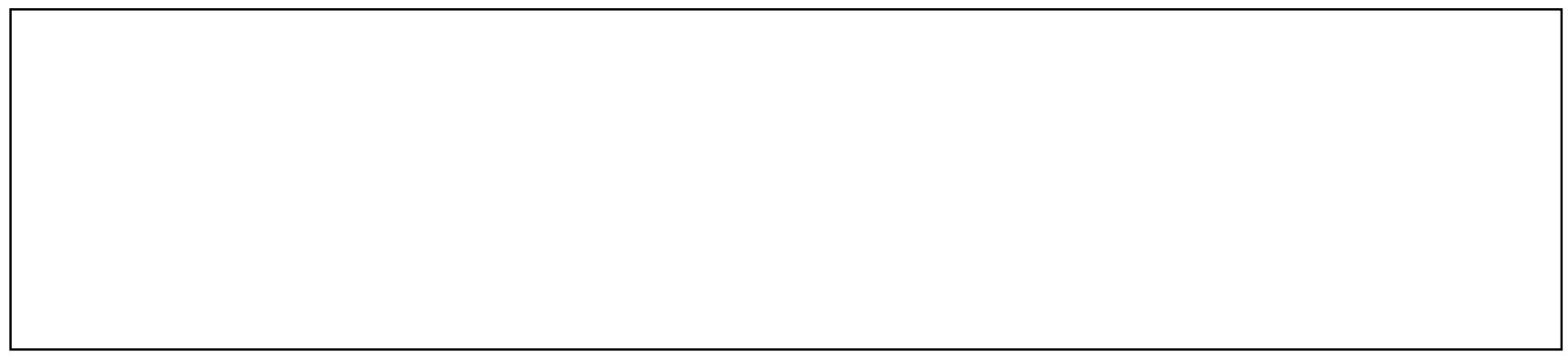




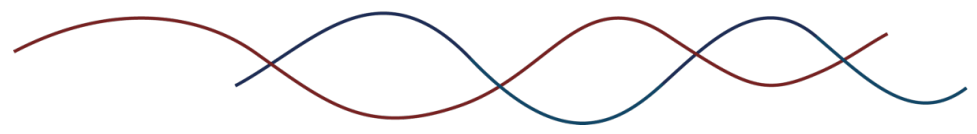

$11^{\text {th }}$ January 2021

Dear Editor,

Please find enclosed our revised manuscript entitled "Molecular determinants of binding of nonoxime bispyridinium nerve agent antidote compounds to the adult muscle nAChR" for consideration for publication in Toxicology Letters.

We thank the Reviewers for their comments and consideration of the manuscript. We have addressed all of the comments and attached a point-by-point response to the comments along with a highlight version of the manuscript for convenience of the review process.

Please let us know if there is anything else needed.

Yours faithfully,

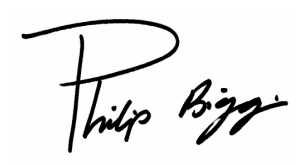

Professor Philip Biggin. CChem. FRSC. 